\title{
Getting Drowsy? Alert/Nonalert Transitions and Visual Thalamocortical Network Dynamics
}

\author{
Yulia Bereshpolova, ${ }^{1}$ Carl R. Stoelzel, ${ }^{1}$ Jun Zhuang, ${ }^{1}$ Yael Amitai, ${ }^{2}$ Jose-Manuel Alonso, ${ }^{1,3}$ and Harvey A. Swadlow ${ }^{1,3}$ \\ ${ }^{1}$ Department of Psychology, University of Connecticut, Storrs, Connecticut 06269, 2Department of Physiology, Ben-Gurion University, Beer-Sheva 84105, \\ Israel, ${ }^{3}$ Department of Biological Sciences, State University of New York, New York, New York 10036
}

The effects of different EEG brain states on spontaneous firing of cortical populations are not well understood. Such state shifts may occur frequently under natural conditions, and baseline firing patterns can impact neural coding (e.g., signal-to-noise ratios, sparseness of coding). Here, we examine the effects of spontaneous transitions from alert to nonalert awake EEG states in the rabbit visual cortex ( $5 \mathrm{~s}$ before and after the state-shifts). In layer 4, we examined putative spiny neurons and fast-spike GABAergic interneurons; in layer 5, we examined corticotectal neurons. We also examined the behavior of retinotopically aligned dorsal lateral geniculate nucleus (LGNd) neurons, usually recorded simultaneously with the above cortical populations. Despite markedly reduced firing and sharply increased bursting in the LGNd neurons following the transition to the nonalert state, little change occurred in the spiny neurons of layer 4. However, fast-spike neurons of layer 4 showed a paradoxical increase in firing rates as thalamic drive decreased in the nonalert state, even though some of these cells received potent monosynaptic input from the same LGNd neurons whose rates were reduced. The firing rates of corticotectal neurons of layer 5, similarly to spiny cells of layer 4, were not state-dependent, but these cells did become more bursty in the nonalert state, as did the fast-spike cells. These results show that spontaneous firing rates of midlayer spiny populations are remarkably conserved following the shift from alert to nonalert states, despite marked reductions in excitatory thalamic drive and increased activity in local fast-spike inhibitory interneurons.

\section{Introduction}

Baseline firing rates and interspike interval (ISI) distributions can influence neuronal computations, coding, and synaptic dynamics (Softky and Koch, 1993; Swadlow and Gusev, 2002; Hahnloser et al., 2003; Castro-Alamancos, 2004). Firing rates are generally higher in awake than in anesthetized subjects (Chen et al., 2009), but wakefulness is not a unitary state and the effects of different awake states on the activity of cortical subpopulations are relatively unstudied. One problem has been the lack of an accepted taxonomy for awake substates. For example, fast-spike interneurons in layer $2 / 3$ of mouse barrel cortex decrease their firing rates following the transition from quiet wakefulness to an active brain state that is associated with whisking and cortical desynchronization (Gentet et al., 2010). In contrast, putative fast-spike interneurons in the visual cortex dramatically increase their firing rates when mice transition from quietly sitting on a sphere to active locomotion (Niell and Stryker, 2010). These interesting, but seemingly contrasting, results are difficult to reconcile because the brain states in these studies are not necessarily the same,

Received May 5, 2011; revised Sept. 12, 2011; accepted 0ct. 12, 2011.

Author contributions: Y.B., C.R.S., Y.A., J.-M.A., and H.A.S. designed research; Y.B., C.R.S., J.Z., Y.A., and H.A.S. performed research; Y.B., C.R.S., Y.A., J.-M.A., and H.A.S. analyzed data; Y.B., C.R.S., Y.A., J.-M.A., and H.A.S. wrote the paper.

This work was supported by National Institutes of Health Grant EY018251.

Correspondence should be addressed to Harvey A. Swadlow, Department of Psychology (U-1020), The University of Connecticut, 406 Babbidge Road, U-1020, Storrs, CT 06269. E-mail: harvey.swadlow@uconn.edu.

DOI:10.1523/JNEUROSCI.2262-11.2011

Copyright $\odot 2011$ the authors $\quad 0270-6474 / 11 / 3117480-08 \$ 15.00 / 0$ the relationship between them is not clear, and each is associated with different behaviors.

Here, we examine spontaneous firing rates and ISI distributions in the visual cortex of awake rabbits as they switch between alert and nonalert awake brain states [defined by hippocampal EEG activity (Green and Arduini, 1954)]. We focused on the alert to nonalert transition ( $5 \mathrm{~s}$ before and after) to assure that the change was not elicited by uncontrolled sensory or motor events. This state transition is often rapid ( $<1 \mathrm{~s})$ and, in thalamocortical neurons, is associated with sharply reduced firing rates and irregular and bursty spike trains (Swadlow and Gusev, 2001; Bezdudnaya et al., 2006; Stoelzel et al., 2008). We studied the effects of this state transition on spontaneous activity of neurons in the primary input layer of visual cortex, layer 4 , and a primary output layer, layer 5. In layer 4, we identified putative spiny neurons (regular spiking neurons with simple receptive fields) as well as putative fast-spike neurons [suspected inhibitory interneurons (SINs) with ON-OFF, nonoriented receptive fields (Swadlow and Weyand, 1987; Swadlow, 1988)]. This latter population receives strong thalamocortical input and provides feedforward inhibition onto the spiny neurons (Swadlow, 2003; Cruikshank et al., 2007). In addition, we simultaneously studied retinotopically aligned neurons in the dorsal lateral geniculate nucleus (LGNd) and corticotectal neurons in layer 5. Remarkably, despite profound state-related changes in firing rates and patterns at the thalamic level, little change was seen in either cortical spiny population. Moreover, layer 4 SINs increased their firing rates in the nonalert state even though they receive strong monosynaptic input from LGNd neurons, whose rates were sharply reduced. 
These results show that the spontaneous activity of midlayer spiny cortical populations is remarkably conserved when subjects shift from an alert to a nonalert state, despite the profound staterelated reductions in excitatory drive from the thalamus and the increased activity in fast-spike interneurons that provide feedforward inhibition.

\section{Materials and Methods}

Recordings were obtained from monocular primary visual cortex (V1) of four awake adult female Dutch-Belted rabbits. The general surgical procedures for chronic recordings have been described previously (Swadlow, 1991; Swadlow et al., 1998) and are reported only briefly here. All experiments were conducted with the approval of the University of Connecticut Animal Care and Use Committee in accordance with National Institutes of Health guidelines.

Animal preparation. Initial surgery was performed under ketamineacepromazyne anesthesia using aseptic procedures. After removal of the skin and fascia, stainless steel screws were installed on the dorsal surface of the skull and fused together with acrylic cement. A stainless steel rod, oriented in a rostrocaudal direction, was then cemented to the acrylic mass. The rabbit was held rigidly by this rod during the electrode implantation and recording sessions. Silicone rubber was used to buffer the wound margins from the acrylic cement on the skull. Following at least $10 \mathrm{~d}$ of recovery, recordings of neuronal activity were obtained in the awake state through a small hole in the skull.

Recording and electrophysiological signal acquisition. Recordings were obtained from V1 and, in some cases, simultaneously from a topographically aligned region of the LGNd of awake rabbits. For cortical singleunit recordings, a concentric array of seven independently controlled electrodes (Swadlow et al., 2005) was chronically implanted within monocular region of V1. The microelectrodes were built from $40 \mu \mathrm{m}$ quartzinsulated, platinum-iridium filaments that were pulled and ground to a fine tip (impedance, 1.5-3 MOhm). Thalamic activity was recorded using the same seven-electrode system with electrodes placed within the LGNd. In another set of experiments, cortical recordings were obtained using a 16-channel vertical probe (NeuroNexus Technologies) from a topographically aligned region of $\mathrm{V} 1$ following mapping procedures (Bereshpolova et al., 2007). Spike waveforms were initially identified during the experiment and verified off-line for each cell using Plexon cluster analysis software. For identification of cortical neurons, two to four stimulating electrodes were implanted within the LGNd and superior colliculus. Sparse noise stimulation and reverse correlation method were applied for determination of visual receptive fields. The receptive fields were calculated in spikes per second, normalized by the response peak, and transformed into contour plots that were smoothed using bicubic interpolation. Identification of layer 4 was based on the reversal point of the field potential generated by a diffuse flash stimulus. Hippocampal EEG was recorded with platinum-iridium microwires. Neocortical EEG was also recorded using a 16-channel vertical probe (NeuroNexus probe sites separated by $100 \mu \mathrm{m}$ ) within the visual cortex during the study of all but two of the neurons recorded herein.

Cell identification. We identified LGNd thalamocortical neurons using spike-triggered averages of axonal field potentials elicited in layers 4 and 6 of the retinotopically aligned region of V1 (Swadlow et al., 2002; Jin et al., 2008; Stoelzel et al., 2008). Putative fast-spike interneurons (SINs) of layer 4 were identified by a high-frequency $(>600 \mathrm{~Hz})$ burst of three or more spikes elicited by electrical stimulation of afferent pathways. All interneurons studied here responded to LGN stimulation at very short latencies ( $<2 \mathrm{~ms}$ ) with spikes of $<0.6 \mathrm{~ms}$ duration (negative plus positive components). The receptive fields of these cells all showed a strong spatial overlap of ON and OFF subregions (Fig. $1 A$ ). Putative spiny, regularspiking (RS) neurons of layer 4 were required to meet both of the following criteria: (1) a spike duration (initial negative wave plus the secondary positive wave) of $>0.6 \mathrm{~ms}$ and (2) a simple receptive field showing orientation selectivity and one, two, or three spatially separate $\mathrm{ON}$ and/or OFF subfields (Fig. $1 A$ ). Corticotectal neurons were identified by their antidromic activation following electrical stimulation of the superior colliculus.

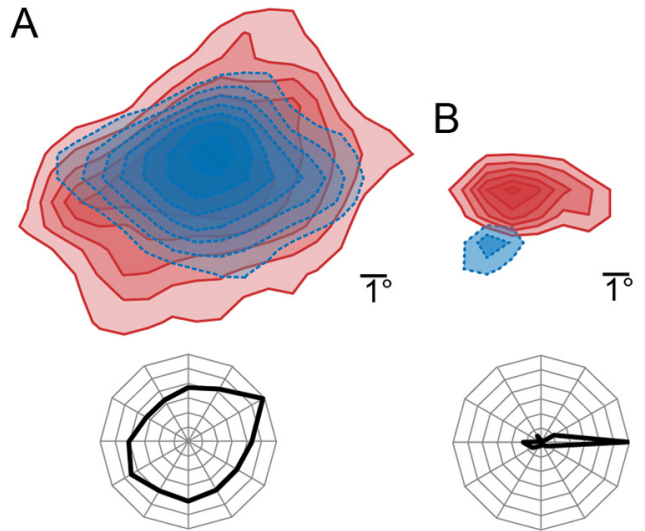

Figure 1. Receptive fields of putative spiny neurons and SINs of layer 4.A, An example of the receptive field of SIN, constructed using the reverse correlation method from response to sparse noise stimulation. The receptive field of SIN has spatially overlapping ON (red) and OFF (blue) subregions (top). In contrast to RS simple cells, SINs are either not sensitive to orientation or exhibit only a weak orientation bias (bottom). $\boldsymbol{B}$, The receptive field of a layer 4 RS simple cell has a strong ON (red) subregion and a small and weaker OFF (blue) region (top). These cells show high orientation selectivity to drifting gratings (bottom).

In a limited number of cases, cross-correlation analysis was used to infer monosynaptic connectivity between LGNd neurons and visual cortical SINs. These studies require precise retinotopic alignment between thalamic and cortical recording sites. When this was achieved, crosscorrelograms, based on the spontaneous firing of LGNd neurons and SINs, were computed. Monosynaptic connectivity was inferred from the presence of significant peaks in the probability of SIN firing at intervals of 1.1-2.5 ms following thalamic spikes. A peak in a cross-correlogram was defined as significant when at least two of three successive bins $(0.1 \mathrm{~ms}$ bin width) in the peak exceeded the 0.01 confidence level. Baseline firing was calculated between -4 and $+1 \mathrm{~ms}$ of the time of thalamic firing (Swadlow and Gusev, 2001, 2002).

EEG states. Once cells had been identified, as described above, and receptive fields and responses to electrical stimulation had been characterized, long periods ensued during which the receptive field and surrounding region of visual space received a constant illumination provided by a blank CRT monitor, which covered $30 \times 22$ degrees of visual space. Hippocampal EEG was monitored while neurons fired spontaneously. The EEG was segmented by visual inspection into alert versus nonalert states based on the presence of theta activity $(5-7 \mathrm{~Hz})$ or high-voltage, irregular activity, respectively (Swadlow and Gusev, 2001; Bezdudnaya et al., 2006; Stoelzel et al., 2008). This segmentation was aided and verified by fast Fourier transform (FFT) analysis. We also recorded neocortical EEG during the study of most of the neurons. In some cases, when rabbits became drowsy during extended recordings, we provided novel sounds to generate alertness and studied the transitions back to a nonalert state.

Our main analyses were restricted to $5 \mathrm{~s}$ periods before and after the EEG transitioned spontaneously and sharply from hippocampal theta activity (alert) to high-voltage irregular activity (nonalert). We did not study the reverse transitions (from nonalert to alert state) because some such transitions could be associated with extraneous noises or selfgenerated movements. The transition from alert to nonalert state could not be due to such factors. Unless otherwise stated, the awake, alert state was defined by a predominance of hippocampal theta activity $(5-7 \mathrm{~Hz}$ range; see below) that occurred just before a transition to non-theta activity. The awake but nonalert state was defined as the $5 \mathrm{~s}$ period immediately after the termination of such theta activity when the hippocampus switches to high-voltage irregular activity. A representative hippocampal EEG trace, with a transition from the alert to the nonalert state is shown in Figure $2 \mathrm{~A}$. For all cells studied in both alert and nonalert states, the average of the power spectral density functions of hippocampal EEG was measured in each state (Fig. 2 B). During nonalert periods, we observed a greatly reduced power in the theta frequency and an in- 
creased power in lower $(1-4 \mathrm{~Hz})$ frequencies. Neocortical EEG provided confirmation of our hippocampal indices of state. We always observed that desynchronized neocortical EEG characterized the alert period, and this transitioned to slow-wave activity during the nonalert period (Fig. 2A).

In addition to the fact that the $5 \mathrm{~s}$ nonalert period studied here immediately followed an alert, vigilant state, a number of factors make us confident that this period represents an awake, but nonalert (or drowsy) state, not an early stage of sleep. First, we never observed sleep spindles in the neocortical EEG during this period ( $>9005 \mathrm{~s}$ periods examined), and in rodents, sleep spindles are widely viewed as indicative of the early stages of sleep (Johns et al., 1977; Gervasoni et al., 2004). Second, the eye remained open across this transition. We used an optical eye tracking system (Arrington Research) to measure eyelid positions during 50 such transitions (measures of the distance between upper and lower lid taken $4 \mathrm{~s}$ before and $4 \mathrm{~s}$ after the transition) and found a mean lid closure of $<4 \%$. Finally, during this period, rabbits were very responsive to innocuous novel visual (waving hand, moving spot of light on ceiling) or other stimulation, readily shifting back to the alert (theta) state.

In addition to being present in alert rabbits, hippocampal theta activity also occurs during REM sleep. However, during REM, it occurs at higher frequencies $(7-8 \mathrm{~Hz})$ (Harper, 1971; Tobler et al., 1990) than during awake vigilance. Notably, the 5-7 Hz hippocampal theta activity that we used to define the $5 \mathrm{~s}$ alert periods just before transitions to nonalert periods showed, for all of the neurons studied here, peak frequencies of $5.25-6.25 \mathrm{~Hz}$ (Fig. 2 B,C). For a variety of reasons, we sometimes allowed rabbits to enter a sleep cycle, and bouts of neocortical spindling and REM sleep sometimes occurred. Figure $2 C$, open bars, shows the peak theta frequencies during several REM bouts that we observed in one rabbit, each of which was marked by twitching of whiskers and eyelids and partial closure of the eye. Importantly, there is no overlap of the theta frequencies seen during these REM bouts (mean, $8.0 \pm 0.13 \mathrm{~Hz}$ ) with those seen during the periods of alert wakefulness (Fig. $2 C$, filled bars; mean, $5.73 \pm 0.03 \mathrm{~Hz}$ ) when our neurons were studied.

\section{Results}

Data presented here were recorded from 24 LGNd neurons and 41 identified cortical neurons (10 layer 4 RS simple cells, 15 layer 4 SINs, and 16 corticotectal neurons) from four rabbits. The spontaneous activity of each of these cells was studied during $5 \mathrm{~s}$ alert and nonalert periods, before and after the transition between alert and nonalert states. Twelve to 45 such transitions were analyzed for each of the neurons that were studied.

\section{Spontaneous activity of LGNd and cortical neurons during alert and nonalert states}

The average firing rate of neurons studied in both alert and nonalert states is shown in Figure $3 \mathrm{~A}$. Each point reflects, for a single neuron, the average firing rate in each state for all of the $5 \mathrm{~s}$ periods before and after the many state transitions that were studied for that cell. Figure $3 B$ summarizes the population responses to state changes in the four cell types studied. LGNd neurons discharged at $11.8 \pm 1.4 \mathrm{~Hz}$ during the alert period and this rate was reduced by $36 \%$ to $7.8 \pm 1 \mathrm{~Hz}$ (paired $t$ test, $p<0.001$ ) following the transition to the nonalert state. Notably, RS simple cells of layer 4, which receive strong input from LGNd neurons (Peters and Payne, 1993; Ahmed et al., 1994), did not mirror the change seen in the LGNd cells and were little affected by state. RS simple cells fired at $1.3 \pm 0.4 \mathrm{~Hz}$ when alert and $1.5 \pm 0.4 \mathrm{~Hz}$ when not alert. The firing rates of corticotectal neurons also showed little effect of state, firing at $6.6 \pm 1.7 \mathrm{~Hz}$ when alert and $8.0 \pm 2.5 \mathrm{~Hz}$ when not alert. Neither of these results were statistically significant (paired $t$ test, RS simple cell, $p=0.16$; corticotectal, $p=0.13$ ). In contrast to RS simple and corticotectal cells, SINs, which receive very powerful input from LGNd cells (Freund et al., 1985; Gibson et al., 1999), showed, paradoxically, a $44 \%$ increase in their spontaneous firing rates during nonalert states $(11.6 \pm 3.3$ and $16.7 \pm 4.5 \mathrm{~Hz}$ in alert and nonalert states, respectively; paired $t$ test; $p<0.001$ ). Indeed, 14 of 15 SINs showed a higher firing rate during the nonalert periods than during the alert periods.

\section{Reliability of responses for individual cells}

The data presented in Figure $3 A$ reflect the averages for all of the state transitions undergone by each cell studied. We also examined the reliability of the responses of single cells. For each of the 14 SINs that showed an average increase in the firing rate during the nonalert state, a within-cell statistical analysis was calculated by comparing repeated transitions that each cell made from alert to nonalert state (12-42 transitions for the different SINs, mean number of transitions $=23$ ); the increased firing rate in the nonalert state was statistically significant for each of these cells (paired $t$ test, $p<0.001$ ). Interestingly, the state-related change in firing rate for the single SIN that reduced its firing rate in the 


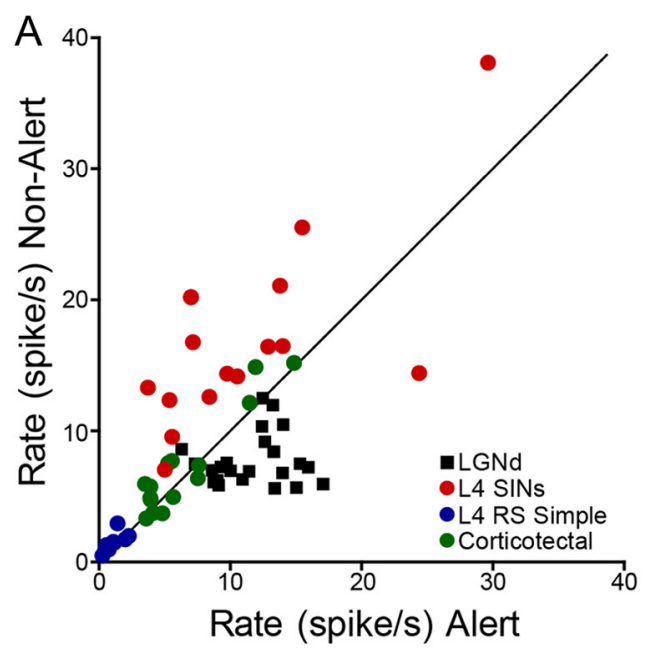

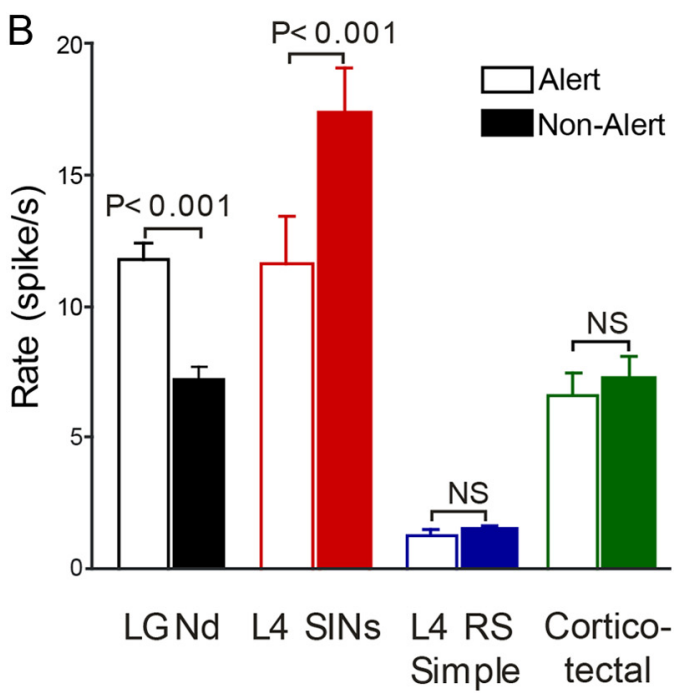

Figure 3. Spontaneous firing rates of LGNd and cortical neurons during alert and nonalert states. $A$, Each data point represents the mean firing rate across all state transitions of a single neuron of the different classes studied in both alert and nonalert states. $\boldsymbol{B}$, The population average of spontaneous firing rates for each class of neurons studied during different states of vigilance.

nonalert state was also highly significant $(p<0.001)$. This same within-cell analysis was performed on LGNd cells and, for 22 of the 24 cells shown in Figure $3 A$, the state-related changes in firing rate were significant $(p<0.01)$.

In some experiments, we recorded simultaneous activity of several neurons across multiple state shifts. Figure $4 A$ presents an example in which two LGNd neurons and two retinotopically aligned SINs were studied simultaneously across 22 state shifts. Our example shows that both LGNd neurons fired at consistently lower rates in the nonalert state, and both SINs fired at consistently higher rates (paired $t$ tests, $p<0.001$ ). Figure $4 B$ shows another example, in which an LGNd neuron, a layer 4 RS simple cell, and a corticotectal neuron were studied simultaneously across 34 state shifts. Whereas the LGNd neuron had a consistently reduced firing rate in the nonalert state, neither of the cortical neurons did.

State-dependent changes in firing frequency among synaptically connected pairs of neurons

Layer 4 SINs of visual cortex receive a potent excitatory input from topographically aligned thalamocortical neurons, and cor-
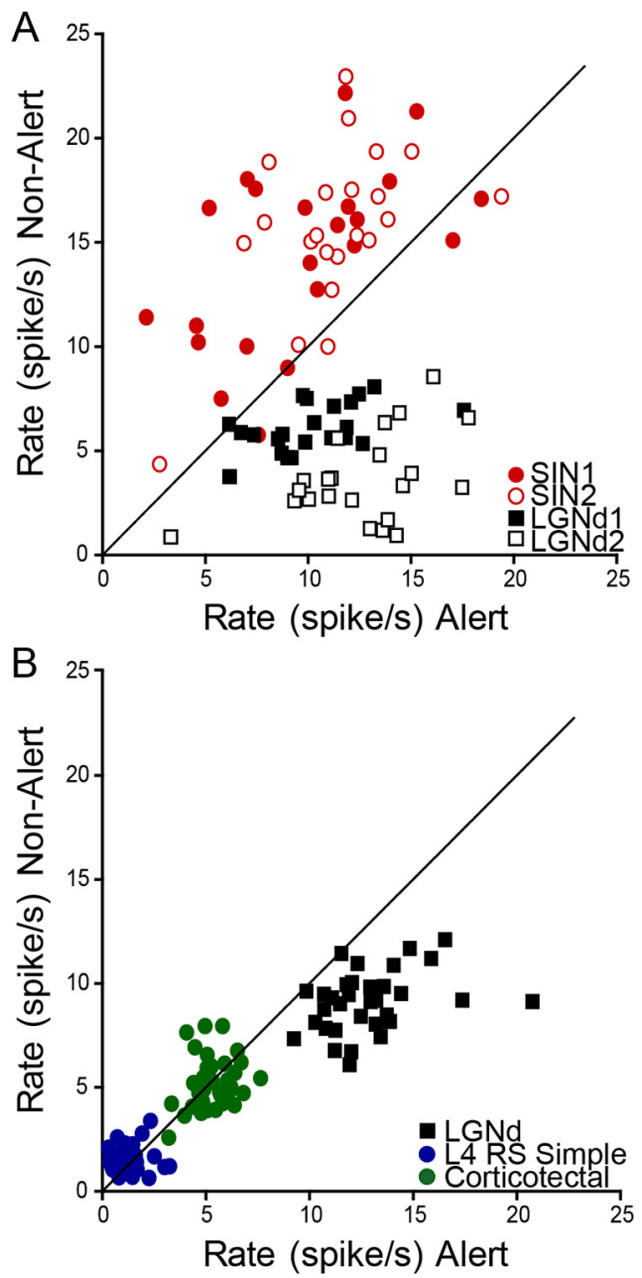

Figure 4. Within-cell consistency of responses to state transitions. $\boldsymbol{A}$, Relation between state and spontaneous firing rate in simultaneously recorded neurons studied during repeated transitions from alert to nonalert state. Two LGNd cells (black open and filled squares) and two SINs (red open and filled circles) underwent 22 shifts from alert to nonalert states, and each data point represents the spontaneous activity during the $5 \mathrm{~s}$ before and $5 \mathrm{~s}$ after each of these shifts. $\boldsymbol{B}$, Another example, where simultaneous recordings were obtained from an LGNd neuron (black squares), a corticotectal neuron (green circles) and a layer $4 \mathrm{RS}$ simple cell (blue circles). These cells underwent 34 state shifts from alert to nonalert states.

tical and thalamic cell pairs that are monosynaptically connected can be reliably identified in vivo by cross-correlation analysis (Swadlow, 1995; Alonso et al., 1996; Usrey et al., 1999; Swadlow and Gusev, 2001). Thus, when we simultaneously recorded LGNd and cortical neurons that were in retinotopic alignment, we reviewed the cross-correlograms of spontaneous spikes for every possible pair to reveal these connections. In seven recordings, we examined the state-related changes in firing frequency among monosynaptically connected pairs (see Materials and Methods, above) of LGNd neurons and V1 SINs in layer 4. As described above, these two classes of neurons exhibited very different patterns of state-related modulation of their firing rates. Even in cases where we were able to observe a dramatic reduction in firing during the nonalert state for an identified LGNd cell, the postsynaptic layer 4 SIN showed significant and reliable increases in spontaneous firing. Examples of two representative cases are shown in Figure 5. In the first (Fig. 5A1,B1,C1), a single LGNd neuron is recorded with two retinotopically aligned SINs (Fig. $5 A 1)$ and cross-correlation of spontaneous spike trains reveals brief, significant $(p<0.001)$ peaks in the spike probabilities of 
the SINs following the LGNd spikes, indicative of synaptic connectivity (Swadlow and Gusev, 2001). Nevertheless, as LGNd firing rate decreases in the nonalert state, SIN firing rates increase (Fig. 1C1). Figure 5, $A 2, B 2$, and $C 2$, shows a second case where an LGNd neuron was recorded with a single retinotopically aligned and monosynaptically connected SIN.

\section{Firing patterns in different cell types} during alert and nonalert states We next analyzed the state-dependence of the ISI distributions for each of the studied cell classes by comparing the $5 \mathrm{~s}$ periods before and after state transitions (Fig. $6 A$ ). Bursting LGNd neurons showed a bimodal distribution where the first peak contained the brief ISIs occurring within the bursts. All recorded LGNd cells showed high levels of bursting in the nonalert state compared with the alert state, with the first peak occurring at an interval of $\sim 5$ ms. Most SINs (12 of 15 neurons) also showed a considerably higher degree of short ISIs following the state transition to the nonalert. However, for SINs, the first peak occurred at intervals of $14 \pm 3$ ms. Notably, RS simple cells of layer 4 and corticotectal cells showed little highfrequency responding in either state. Figure $6 B$ shows the same analysis, but for the much larger dataset consisting of all periods when the animal was alert or nonalert, based on the EEG (not only the $5 \mathrm{~s}$ periods around the state changes). Interestingly, these distributions appear very similar to those in Figure $6 \mathrm{~A}$ but are, of course, smoother.

To examine this question further, we first defined bursts in cortical neurons according to criteria (Fig. 7A) often applied to thalamic neurons (Sherman and Guillery, 1996; Bezdudnaya et al., 2006). Here, bursts were identified as clusters of two or more spikes with ISIs of $\leq 4 \mathrm{~ms}$, where the initial spike of the burst had a preceding interval of at least $100 \mathrm{~ms}$. We measured the proportion of cortical spikes that participate in bursts (the burst fraction). We found all cortical cell types showed higher bursting rate during the nonalert state compared with the alert state. This is statistically significant for SINs and corticotectal neurons (Wilcoxon test, $p<0.001$, corrected for multiple comparisons using Bonferroni adjusted alpha levels) (Dunn, 1961), though bursting is considerably more pronounced in LGNd neurons. There was no significant increase in bursting for RS simple cells $(p>0.1)$. Since cortical bursting may not follow the criteria established for the thalamus, we relaxed the ISI requirement for the first spike pair following $100 \mathrm{~ms}$ rest to $\leq 7 \mathrm{~ms}$ (Fig. $7 B$ ) or $\leq 10 \mathrm{~ms}$ (Fig. 7C). Using these relaxed criteria, SINs and corticotectal neurons continued to show a statistically significant effect of state on bursting ( $p<0.001)$, but RS simple cells did not $(p>0.1)$.

As seen above, the increased bursting of LGNd neurons, SINs, and corticotectal neurons that occurred during the $5 \mathrm{~s}$ following the transition to the nonalert state held across a broad parameter range for the definition of a burst. RS simple cells, by contrast,
A2
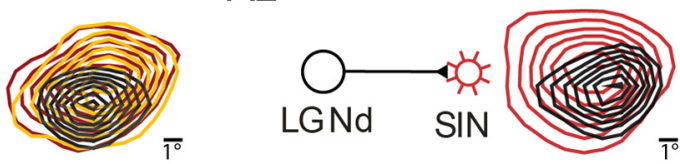

B2

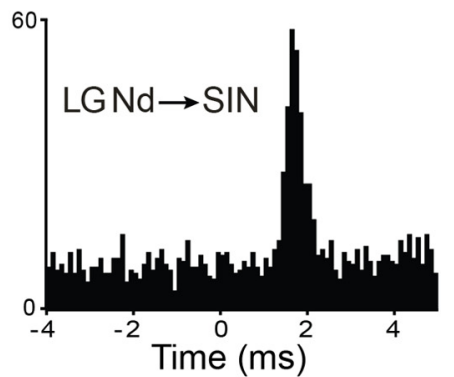

$\mathrm{C} 2$

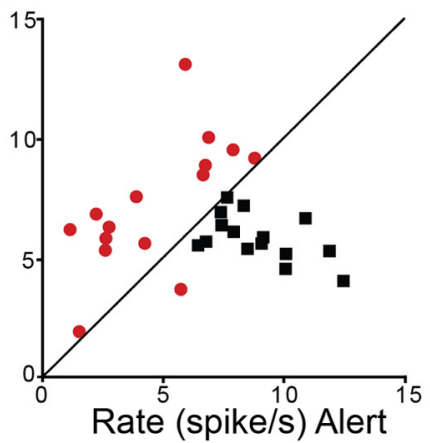

Figure 5. State-dependent opposing shifts in firing frequency between synaptically connected SINs and LGNd neurons. $\boldsymbol{A} \mathbf{1}, \boldsymbol{B} \mathbf{1}$, C1, Spontaneous action potentials of a single LGNd neuron were simultaneously recorded with those of two retinotopically aligned

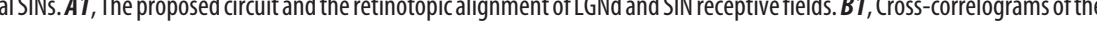
and after alert to nonalert shifts. SINs are shown as open and filled circles, LGNd neurons as black squares. A2, B2, C2, Same as A1, $B 1$, and C1, respectively, but for another LGNd neuron and a monosynaptically connected V1 SIN.

showed no significant state differences using any of the above definitions. Moreover, we also showed that RS neurons showed no significant state effect on bursting when bursts were required to contain at least three spikes (rather than two), or when they were defined even more loosely, as requiring three successive spikes with a maximal interspike interval of $80 \mathrm{~ms}$ between the two first spikes and a maximum of $160 \mathrm{~ms}$ for all intraburst ISIs (Grace and Bunney, 1984).

\section{Discussion}

Our results show that natural transitions from alert to nonalert states are associated with changes in spontaneous firing rate and ISI distributions that are highly specific to cell type within the thalamocortical networks. Consistent with our previous results for thalamocortical neurons (Swadlow and Gusev, 2001; Bezdudnaya et al., 2006; Stoelzel et al., 2008), LGNd neurons show abrupt reductions in spontaneous firing and increases in bursting when nonalert. Cortical layer 4 RS simple cells, the main recipients of the thalamic input, maintain constant rate of spontaneous spiking activity and ISI distributions throughout state transition. SINs of layer 4, considered the main feedforward inhibitory elements in the circuit, increase their spontaneous firing rate when nonalert, contrary to the tendency of their thalamic drivers, accompanied by minor increases in high-frequency bursts. Finally, corticotectal neurons of layer 5 show no significant state- 
A
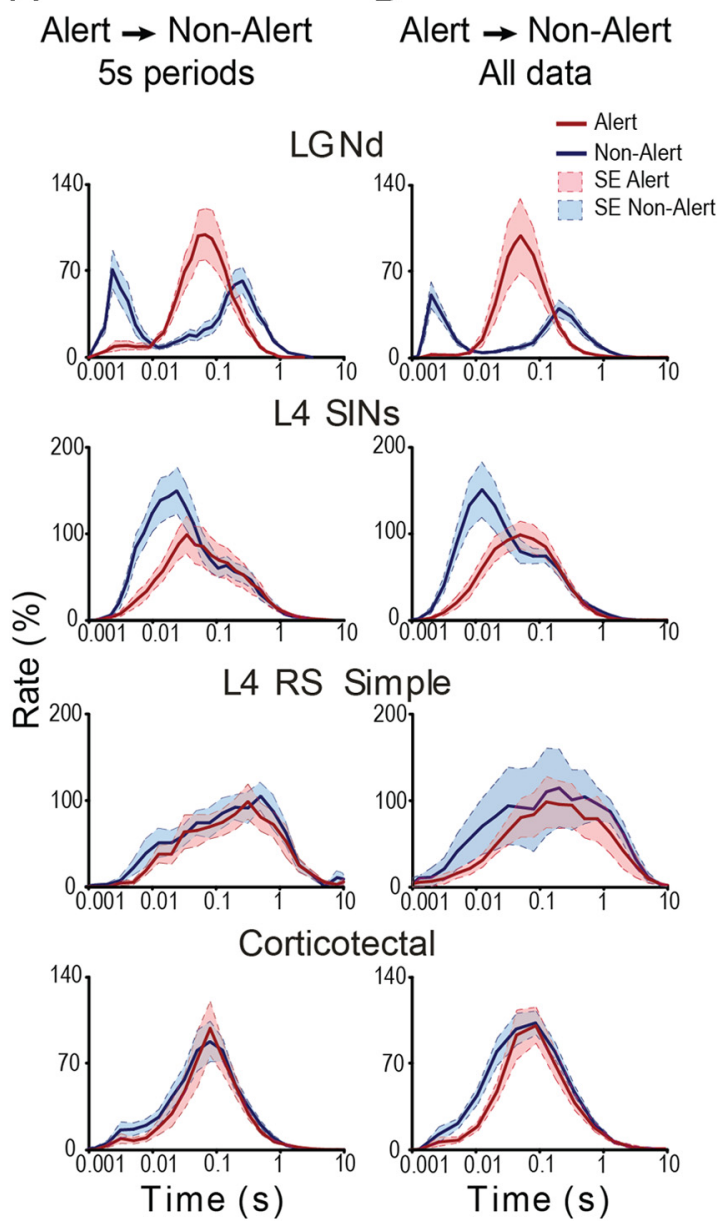

Figure 6. The state-related distribution of interspike intervals in different cell types. $A$, The ISI histograms of different cell classes during alert (red lines) and nonalert (blue lines) epochs. Only the $5 \mathrm{~s}$ periods before and after the transitions from alert to nonalert state are included, and data from all of the cells of each class (normalized) are included. The bimodal shape of the distribution for LGNd cells and SINs reflects the intervals occurring within and between bursts. Alert and nonalert states are shown by solid and dashed lines, respectively. $\boldsymbol{B}$, The same analysis as in $A$, but composed of a much larger dataset that included all alert and nonalert segments during the entire data file. The shaded areas in $\boldsymbol{A}$ and $\boldsymbol{B}$ show the SEM periods.

dependent changes in their spontaneous firing rates, and only a modest increase in bursting when nonalert. This latter finding is notable because the great majority of corticotectal neurons tested in vitro (Kasper et al., 1994; Rumberger et al., 1998; Tsiola et al., 2003) have been classified as "intrinsically bursting" (Connors et al., 1982; McCormick et al., 1985; Chagnac-Amitai and Connors, 1989).

\section{Previous studies of cortical neurons across awake substates}

To the best of our knowledge, this is the first report demonstrating specific conditions of the thalamocortical networks under which cortical neurons do not follow or even move in opposite direction to the trend of thalamic relay neurons, considered their drivers. A considerable amount of previous literature indicates that spontaneous firing of cortical neurons is greater in awake versus anesthetized brains (for recent examples, see Greenberg et al., 2008; Chen et al., 2009; Sakata and Harris, 2009). There is little agreement, however, about activity levels during different awake states. In some studies, spontaneous firing during quiet immobility has been compared with an awake, behaving condition, and
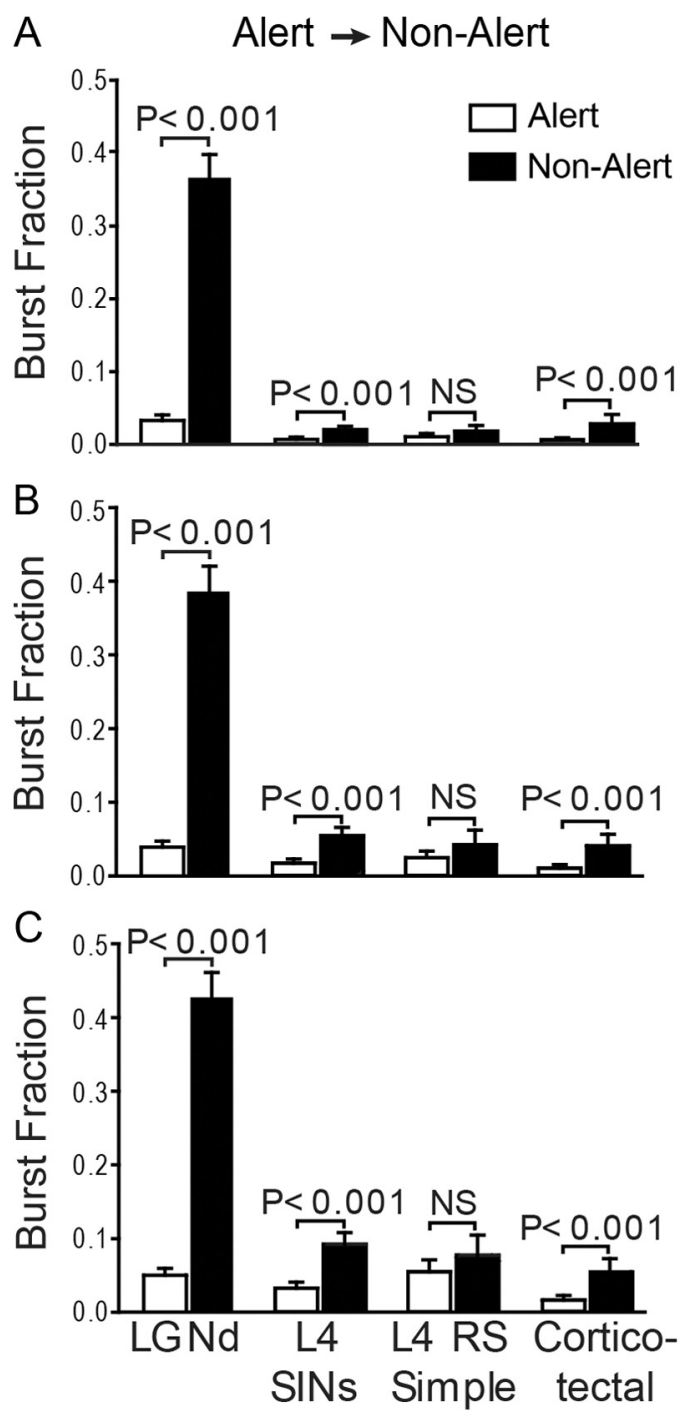

Figure 7. $A-C$, Rapid changes in bursting of LGNd neurons and some cortical populations within $5 \mathrm{~s}$ of a state switch from alert to nonalert. Bars indicate percentage of spikes ( $\pm S E$ ) that are part of the bursts for the different cell classes during alert (open) and nonalert (filled) epochs. Bursts were defined as two or more spikes with a preceding interval $>100 \mathrm{~ms}$ and an ISI of either $\leq 4(\boldsymbol{A}), \leq 7(\boldsymbol{B})$, or $\leq 10(\boldsymbol{C}) \mathrm{ms}$. LGNd cells, SINs, and corticotectal neurons, but not RS simple cells, showed increased bursting in the nonalert state.

diverse results have been obtained. Thus, Buzsaki et al. (1988) found that spontaneous firing in many areas of rat cortex increases during locomotion, compared with a non-locomoting, awake state, but Greenberg et al. (2008) found no movementrelated differences in activity of rat layer $2 / 3$ neurons visualized with two-photon microscopy. Similarly contradictory, Gentet et al. (2010) report that fast-spike interneurons, in superficial layers of barrel cortex, increase their spontaneous firing during quiet wakefulness compared with during whisking (consistent with our results for V1 SINs), but Niell and Stryker (2010) found that fast-spike neurons in mouse visual cortex increase their rate of firing during virtual locomotion, sometimes by $>400 \%$. Such diverse results may be due, in part, to different types of activity that have been measured (e.g., locomotion vs whisking), each of which may be providing different types of sensory input to the cortical areas under study. It is, therefore, often difficult to disentangle brain state from the behaviors associated with these states, and their consequences. In the present study, we mini- 
mized such effects by limiting our analysis to the transition from alert to nonalert EEG state in a subject sitting quietly in both states.

\section{The paradox: deviation from the feedforward model}

It has been widely accepted that thalamic inputs strongly drive neurons in primary sensory cortices during sensory stimuli. Indeed, one might expect, based on a feedforward thalamocortical model, that state-related increases or decreases in the LGNd would be reflected by similar changes in the cortex. While LGNd synapses provide only a small proportion of the synapses onto layer 4 spiny neurons (da Costa and Martin, 2011), thalamocortical synapses are very powerful $[\sim 4.5$ times the strength of intracortical synapses (Gil et al., 1999)] and thalamocortical neurons are much more active than layer 4 spiny neurons (Fig. 3). Thus, thalamocortical synapses provide a far greater synaptic drive onto layer 4 spiny neurons than would be suggested by a simple count of their numbers. However, in contrast to the expectation based on the behavior of LGNd cells, the firing rates of layer 4 spiny neurons were remarkably conserved across states, as was the distribution of their ISIs. This is even more surprising considering the increased activity of layer 4 SINs when nonalert. Furthermore, the potency of fast-spiking inhibitory synapses onto spiny cells is thought to be stronger in the nonaroused state, when acetylcholine levels are low (Kruglikov and Rudy, 2008). Together, in the nonaroused state, these factors (decreased firing of LGNd neurons, increased firing of local feedforward inhibitory neurons, stronger postsynaptic inhibition) would all point to reduced firing in spiny cells, but clearly, this does not happen.

Equally surprising is the case of layer 4 SINs: they increased their activity in the nonalert state, as LGNd firing decreased, and this was shown to be highly reliable in individual neurons over time. Thalamocortical synapses onto fast-spike interneurons are even more powerful than those onto spiny cells of layer 4 (Gibson and Connors, 1999; Cruikshank et al., 2007; Hull et al., 2009). Here, we showed that even those SINs receiving powerful input from an LGNd neuron (Fig. 5) increased their firing rates in the nonalert state, even though that same LGNd neuron showed a markedly decreased rate. These seemingly paradoxical findings cannot be readily explained by a feedforward model of thalamocortical processing.

Possible mechanisms behind thalamocortical disengagement Multiple mechanisms could combine to reduce the effect of the thalamic drive on cortical neurons and account for the findings above. First, thalamocortical synapses are depressing (Gil et al., 1997) and, in vivo, thalamocortical synapses are in a state of chronic synaptic depression because of the high rates of firing (Swadlow and Gusev, 2001; Castro-Alamancos, 2004; Stoelzel et al., 2008). Lower rates of thalamic firing in the nonalert state yield relief from this depression, which could compensate for changes in thalamocortical firing rates, adding to the stability of baseline rates in spiny cells. Second, changing levels of neuromodulators with states are likely to modify the relative impact of network components. Especially studied are the effects of acetylcholine. Layer 4 spiny neurons are hyperpolarized by application of acetylcholine (Eggermann and Feldmeyer, 2009). Thus, in the aroused state, when acetylcholine levels are up, the increased feedforward thalamocortical excitation of layer 4 spiny neurons (and reduced feedforward inhibition) could be countered by this near simultaneous hyperpolarizing current. However, somatostatin-positive GABAergic interneurons are excited by cholinergic inputs (Fanselow et al., 2008) and provide an inhibi- tory synaptic drive onto fast-spike interneurons (Kisvárday et al., 1993; Hughes et al., 2000). Thus, activation of this class of GABAergic interneuron in the alert state would inhibit the SINs, while in the nonalert state they would be disinhibited. Third, although reduced in rate, the firing of LGNd neurons is more bursty when nonalert, and the enhanced effects of bursts on postsynaptic spiking (Lisman, 1997; Sherman, 2001; Swadlow and Gusev, 2001) could have a compensating effect on the spiking of recipient spiny cells. Bursting thalamocortical neurons could also strongly activate cortical SINs, in some cases, generating multiple action potentials (Swadlow and Gusev, 2001). This could increase the overall number of SIN spikes in the cortex despite the reduction in overall firing rate of thalamocortical neurons in the nonalert state.

\section{The significance of getting drowsy}

Considerable advances have been made in our understanding of mechanisms of visual attention in the awake, alert brain (Desimone and Duncan, 1995; Kastner and Ungerleider, 2000; Reynolds and Chelazzi, 2004; Maunsell and Treue, 2006). However, awake subjects are not always alert and attentive. Indeed, many mammals (including humans) are frequently nonalert in familiar environments, but they still must respond appropriately to unexpected changes in their surroundings. "Drowsy driving" kills $>1000$ people and causes tens of thousands of injuries each year in the United States alone [National Highway Traffic Safety Administration (2011). National Highway traffic safety administration review on drowsy driving, http://www.nhtsa.gov/people/ injury/drowsy_driving1/drowsy.html] Drowsiness has been linked to a reduction in the useful visual field (Rogé et al., 2002); however, we still have a poor understanding of how cortical processing is affected by reduced alertness in awake subjects, and quantitative studies of sensory processing have been performed almost exclusively in subjects that are either anesthetized or alert and attentive.

Here, we investigated how reduced alertness affects activity in the primary input layer of the visual cortex and in layer 5 corticotectal output neurons. We demonstrated that, as alertness wanes, a reduced and bursty thalamic output is hardly transmitted to cortical neurons. Remarkably, the baseline rates of layer 4 excitatory neurons and corticotectal neurons remained nearly constant. This consistency in the firing rates of layer 4 spiny neurons may serve to stabilize the input circuit during the transition to drowsiness, so that it remains ready to process the synchronized thalamic activity that can result from sensory stimulation in the nonalert state (Sherman, 2001; Swadlow and Gusev, 2001).

\section{References}

Ahmed B, Anderson JC, Douglas RJ, Martin KA, Nelson JC (1994) Polyneuronal innervation of spiny stellate neurons in cat visual cortex. J Comp Neurol 341:39-49.

Alonso JM, Usrey WM, Reid RC (1996) Precisely correlated firing in cells of the lateral geniculate nucleus. Nature 383:815-819.

Bereshpolova Y, Amitai Y, Gusev AG, Stoelzel CR, Swadlow HA (2007) Dendritic backpropagation and the state of the awake neocortex. J Neurosci 27:9392-9399.

Bezdudnaya T, Cano M, Bereshpolova Y, Stoelzel CR, Alonso JM, Swadlow HA (2006) Thalamic burst mode and inattention in the awake LGNd. Neuron 49:421-432.

Buzsaki G, Bickford RG, Ponomareff G, Thal LJ, Mandel R, Gage FH (1988) Nucleus basalis and thalamic control of neocortical activity in the freely moving rat. J Neurosci 8:4007-4026.

Castro-Alamancos MA (2004) Dynamics of sensory thalamocortical synaptic networks during information processing states. Prog Neurobiol 74:213-247. 
Chagnac-Amitai Y, Connors BW (1989) Synchronized excitation and inhibition driven by intrinsically bursting neurons in neocortex. J Neurophysiol 62:1149-1162.

Chen Y, Anand S, Martinez-Conde S, Macknik SL, Bereshpolova Y, Swadlow HA, Alonso JM (2009) The linearity and selectivity of neuronal responses in awake visual cortex. J Vis 9:12..1-12.17.

Connors BW, Gutnick MJ, Prince DA (1982) Electrophysiological properties of neocortical neurons in vitro. J Neurophysiol 48:1302-1320.

Cruikshank SJ, Lewis TJ, Connors BW (2007) Synaptic basis for intense thalamocortical activation of feedforward inhibitory cells in neocortex. Nat Neurosci 10:462-468.

da Costa NM, Martin KA (2011) How thalamus connects to spiny stellate cells in the cat's visual cortex. J Neurosci 31:2925-2937.

Desimone R, Duncan J (1995) Neural mechanisms of selective visual attention. Annu Rev Neurosci 18:193-222.

Dunn OJ (1961) Multiple comparisons among means. J Am Stat Assoc 56:52-64.

Eggermann E, Feldmeyer D (2009) Cholinergic filtering in the recurrent excitatory microcircuit of cortical layer 4. Proc Natl Acad Sci U S A 106:11753-11758.

Fanselow EE, Richardson KA, Connors BW (2008) Selective, state-dependent activation of somatostatin-expressing inhibitory interneurons in mouse neocortex. J Neurophysiol 100:2640-2652.

Freund TF, Martin KA, Somogyi P, Whitteridge D (1985) Innervation of cat visual areas 17 and 18 by physiologically identified $\mathrm{X}$ - and $\mathrm{Y}$-type thalamic afferents. II. Identification of postsynaptic targets by GABA immunocytochemistry and Golgi impregnation. J Comp Neurol 242:275-291.

Gentet LJ, Avermann M, Matyas F, Staiger JF, Petersen CC (2010) Membrane potential dynamics of gabaergic neurons in the barrel cortex of behaving mice. Neuron 65:422-435.

Gervasoni D, Lin SC, Ribeiro S, Soares ES, Pantoja J, Nicolelis MA (2004) Global forebrain dynamics predict rat behavioral states and their transitions. J Neurosci 24:11137-11147.

Gibson JR, Beierlein M, Connors BW (1999) Two networks of electrically coupled inhibitory neurons in neocortex. Nature 402:75-79.

Gil Z, Connors BW, Amitai Y (1997) Differential regulation of neocortical synapses by neuromodulators and activity. Neuron 19:679-686.

Gil Z, Connors BW, Amitai Y (1999) Efficacy of thalamocortical and intracortical synaptic connections: quanta, innervation, and reliability. Neuron 23:385-397.

Grace AA, Bunney BS (1984) The control of firing pattern in nigral dopamine neurons: burst firing. J Neurosci 4:2877-2890.

Green JD, Arduini AA (1954) Hippocampal electrical activity in arousal. J Neurophysiol 17:533-557.

Greenberg DS, Houweling AR, Kerr JN (2008) Population imaging of ongoing neuronal activityin the visual cortex of awake rats. Nat Neurosci 11:749-751.

Hahnloser RH, Seung HS, Slotine JJ (2003) Permitted and forbidden sets in symmetric threshold-linear networks. Neural Comput 15:621-638.

Harper RM (1971) Frequency changes in hippocampal electrical activity during movement and tonic immobility. Physiol Behav 7:55-58.

Hughes DI, Bannister AP, Pawelzik H, Thomson AM (2000) Double immunofluorescence, peroxidase labelling and ultrastructural analysis of interneurons following prolonged electrophysiological recordings in vitro. J Neurosci Methods 101:107-116.

Hull C, Isaacson JS, Scanziani M (2009) Postsynaptic mechanisms govern the differential excitation of cortical neurons by thalamic inputs. J Neurosci 29:9127-9136.

Jin JZ, Weng C, Yeh CI, Gordon JA, Ruthazer ES, Stryker MP, Swadlow HA, Alonso JM (2008) On and off domains of geniculate afferents in cat primary visual cortex. Nat Neurosci 11:88-94.

Johns TG, Piper DC, James GW, Birtley RD, Fischer M (1977) Automated analysis of sleep in the rat. Electroencephalogr Clin Neurophysiol 43:103-105.

Kasper EM, Larkman AU, Lübke J, Blakemore C (1994) Pyramidal neurons in layer 5 of the rat visual cortex. I. Correlation among cell morphology, intrinsic electrophysiological properties, and axon targets. J Comp Neurol 339:459-474.

Kastner S, Ungerleider LG (2000) Mechanisms of visual attention in the human cortex. Annu Rev Neurosci 23:315-341.

Kisvárday ZF, Beaulieu C, Eysel UT (1993) Network of GABAergic large basket cells in cat visual cortex (area 18): implication for lateral disinhibition. J Comp Neurol 327:398-415.
Kruglikov I, Rudy B (2008) Perisomatic GABA release and thalamocortical integration onto neocortical excitatory cells are regulated by neuromodulators. Neuron 58:911-924.

Lisman JE (1997) Bursts as a unit of neural information: making unreliable synapses reliable. Trends Neurosci 20:38-43.

Maunsell JH, Treue S (2006) Feature-based attention in visual cortex. Trends Neurosci 29:317-322.

McCormick DA, Connors BW, Lighthall JW, Prince DA (1985) Comparative electrophysiology of pyramidal and sparsely spiny stellate neurons of the neocortex. J Neurophysiol 54:782-806.

Niell CM, Stryker MP (2010) Modulation of visual responses by behavioral state in mouse visual cortex. Neuron 65:472-479.

Peters A, Payne BR (1993) Numerical relationships between geniculocortical afferents and pyramidal cell modules in cat primary visual cortex. Cereb Cortex 3:69-78.

Reynolds JH, Chelazzi L (2004) Attentional modulation of visual processing. Annu Rev Neurosci 27:611-647.

Rogé J, Kielbasa L, Muzet A (2002) Deformation of the useful visual field with state of vigilance, task priority, and central task complexity. Percept Mot Skills 95:118-130.

Rumberger A, Schmidt M, Lohmann H, Hoffman KP (1998) Correlation of electrophysiology, morphology, and functions in corticotectal and corticopretectal projection neurons in rat visual cortex. Exp Brain Res 119:375-390.

Sakata S, Harris KD (2009) Laminar structure of spontaneous and sensoryevoked population activity in auditory cortex. Neuron 64:404-418.

Sherman SM (2001) Tonic and burst firing: dual modes of thalamocortical relay. Trends Neurosci 24:122-126.

Sherman SM, Guillery RW (1996) Functional organization of thalamocortical relays. J Neurophysiol 76:1367-1395.

Softky WR, Koch C (1993) The highly irregular firing of cortical cells is inconsistent with temporal integration of random EPSPs. J Neurosci 13:334-350.

Stoelzel CR, Bereshpolova Y, Gusev AG, Swadlow HA (2008) The impact of an LGNd impulse on the awake visual cortex: synaptic dynamics and the sustained/transient distinction. J Neurosci 28:5018-5028.

Swadlow HA (1988) Efferent neurons and suspected interneurons in binocular visual cortex of the awake rabbit: receptive fields and binocular properties. J Neurophysiol 59:1162-1187.

Swadlow HA (1991) Efferent neurons and suspected interneurons in second somatosensory cortex of the awake rabbit: receptive fields and axonal properties. J Neurophysiol 66:1392-1409.

Swadlow HA (1995) The influence of VPM afferents on putative inhibitory interneurons in S1 of the awake rabbit: evidence from cross-correlation, microstimulation, and latencies to peripheral sensory stimulation. J Neurophysiol 73:1584-1599.

Swadlow HA (2003) Fast-spike interneurons and feed-forward inhibition in sensory neocortex. Cereb Cortex 13:25-32.

Swadlow HA, Gusev AG (2001) The impact of "bursting" thalamic impulses on a neocortical synapse. Nat Neurosci 4:402-408.

Swadlow HA, Gusev AG (2002) Receptive-field construction in cortical inhibitory interneurons. Nat Neurosci 5:403-404.

Swadlow HA, Weyand TG (1987) Corticogeniculate neurons, corticotectal neurons, and suspected interneurons in visual cortex of awake rabbits: receptive-field properties, axonal properties, and effects of EEG arousal. J Neurophysiol 57:977-1001

Swadlow HA, Beloozerova IN, Sirota MG (1998) Sharp, local synchrony among putative feed-forward inhibitory interneurons of rabbit somatosensory cortex. J Neurophysiol 79:567-582.

Swadlow HA, Gusev AG, Bezdudnaya T (2002) Activation of a cortical column by a thalamocortical impulse. J Neurosci 22:7766-7773.

Swadlow HA, Bereshpolova Y, Bezdudnaya T, Cano M, Stoelzel CR (2005) A multi-channel, implantable microdrive system for use with sharp, ultrafine "Reitboeck" microelectrodes. J Neurophysiol 93:2959-2965.

Tobler I, Franken P, Scherschlicht R (1990) Sleep and EEG spectra in the rabbit under baseline conditions and following sleep deprivation. Physiol Behav 48:121-129.

Tsiola A, Hamzei-Sichani F, Peterlin Z, Yuste R (2003) Quantitative morphologic classification of layer 5 neurons from mouse primary visual cortex. J Comp Neurol 461:415-428.

Usrey WM, Reppas JB, Reid RC (1999) Specificity and strength of retinogeniculate connections. J Neurophysiol 82:3527-3540. 